\title{
Using molecular docking to find the inhibitor of bacterial cellulose synthesis
}

\author{
I. Petrushin \\ Irkutsk State University, Irkutsk, Russia \\ e-mail:ivan.kiel@gmail.com
}

Key words: molecular docking, biofilm, cellulose synthase

Motivation and Aim: Most of bacterium live into the biofilms. This film protects bacterial colonies against antibiotics and other external impact. It's important to destroy biofilm when treating bacterial infections [1]. Biofilm consist of cellulose, which is resistant to external impact. That's why we chose to block its synthesis by interfering normal work of specific protein - cellulose synthase. Cellulose production begins when small molecule, ligand (c-di-GMP) binds with cellulose synthase. We study how to block active site of cellulose synthase by different ligand.

Methods and Algorithms: We can estimate the energy of interaction between protein and ligand using molecular docking. In this work we use well known AutoDock Vina package to calculate the energy and AutoDock Tools to prepare input files and visualize the results. To narrow the field of search we decided to study group of natural plant metabolites (flavonoids) with protein cellulose synthase which structure and mechanism described in [2]. Flavonoids are commercially available and have antiseptic properties. Results: Virtual screening of hundreds of compounds similar to original ligand (c-diGMP) was studied. We discovered several flavonoids with binding energy closer to energy for original ligand - c-di-GMP.

Conclusion: It's necessary to estimate ligand-protein interaction with molecular dynamics simulation and study ligand impact to biofilm in vitro.

\section{References}

1. Monds R.D., O'Toole G.A. (2009) The developmental model of microbial biofilms: ten years of a paradigm up for review. Trends Microbiology. 17:73-87.

2. Mazur O., Zimmer J. (2011) Apo- and cellopentaose-bound structures of the bacterial cellulose synthase subunit BcsZ. J. Biol. Chem. 286:17601-17606. 